\title{
HIGHER DERIVATIONS OF WILDLY RAMIFIED v-RINGS
}

\section{MARTIN N. HEINZER ${ }^{1}$}

I. Introduction and results. In a number of recent papers Heerema [2], [4], Neggers [6], and the author [5], have used techniques involving the lifting of derivations and (infinite) higher derivations from the residue field $k$, of a local ring $R$, to derivations and higher derivations of $R$. These papers are concerned with the automorphism structure of complete regular local rings. In this paper we will identify the group of higher derivations of $k$ which lift to $R$ when $R$ is a wildly ramified $v$-ring with ramification $p$ over an unramified $v$-subring. In particular let $R_{e}$ be a ramified $v$-ring with ramification $e$. That is, $R_{e}$ is a complete, discrete, rank one, valuation ring having characteristic zero with residue field $k$ of characteristic $p(p$ prime $\neq 2)$ and $p R_{e}$ is the $e$ th power of the maximal ideal $M$ of $R_{e}$. If $e=1$ or $(e, p)=1$ then all higher derivations of $k$ lift to higher derivations of $R_{e}$ [1, Theorem 1, p. 575], [7, Theorem 3.4, p. 24]. We determine which higher derivations of $k$ lift in the simplest wildly ramified case, when $e=p$, and generalize a theorem first proved by Wishart [ 7 , Theorem 3.18 , p. 44] characterizing those rings $R_{p}$ with the property that all higher derivations of $k$ lift.

The symbol $\pi$ will always denote a prime element of $R_{p}$ and $\bar{a}$ the residue of $a \in R_{p}$. We have, $\pi^{p}+p u=0, \bar{u} \neq 0$, and if $\bar{u} \in k^{p}, \pi$ can be chosen so that $\pi^{p}+p\left(1+\pi^{t} w\right)=0, t>0$ and $\bar{w} \neq 0$, or so that $\pi^{p}+p=0$. It is not difficult [5, Lemma 2.3] to see that $\pi$ can be chosen to satisfy precisely one of the following:

$$
\begin{aligned}
\pi^{p}+p u & =0, \quad \bar{u} \notin k^{p} . \\
\pi^{p}+p\left(1+\pi^{t}(\hat{w})\right. & =0, \quad 1 \leqq t \leqq p, \bar{w} \neq 0, \quad \text { and } \quad \bar{w} \notin k^{p}
\end{aligned}
$$$$
\text { when } t=p \text {. }
$$

$$
\pi^{p}+p\left(1+\pi^{p+1} w\right)=0 .
$$

We may identify now two parameters, expo $R_{p}$ and res $R_{p}[5]$, which will have a decisive role in this study.

$$
\begin{aligned}
\operatorname{expo~} R_{p} & =0 & & \text { if } \pi \text { satisfies } 1.1 . \\
& =t & & \text { if } \pi \text { satisfies } 1.2, \mathrm{t} \\
& =p+1 & & \text { if } \pi \text { satisfies } 1.3
\end{aligned}
$$

Received by the editors January 27, 1969.

${ }^{1}$ This research was supported in part by NSF grant GP 8424. 
res $R_{p}=\bar{u} \quad$ if $\pi$ satisfies 1.1 .

$=\bar{r} \quad$ if $\pi$ satisfies $1.2, \mathrm{t}$ or 1.3 and $\bar{r}^{n}=\bar{w}$ with $\bar{r} \notin k^{p}$.

$=0$ otherwise.

Equation 1.5 does not uniquely determine res $R_{p}$. However, res $R_{p}$ is uniquely determined, $\bmod k^{p}$, either with respect to addition or multiplication, and this will be sufficient for our purposes. The quantity expo $R_{p}$ is uniquely determined by 1.4 .

We let $G_{D}$ be the group of derivation automorphisms of $R_{p}$, and $G_{S}$ the group of strongly convergent derivation automorphisms of $R_{p}$ [5]. The symbols $u$ and $w$ will always be used as above.

We now state the results of this study.

THEOREM. The higher derivation $\left\{\delta_{i}\right\}$ of $k$ lifts to a higher derivation of $R_{p}$ if and only if $\delta_{i}$ (res $R_{p}$ ) $=0$ for each $i$ with the following exceptions. If expo $R_{p}=0$ then $\left\{\delta_{i}\right\}$ lifts if and only if (res $\left.R_{p}\right)^{-1} \delta_{i p}\left(\operatorname{res} R_{p}\right) \in k^{p}$ for each $i$ and $\delta_{i}$ (res $R_{p}$ ) $=0$ when $p \nmid i$. If expo $R_{p}=p$ then $\left\{\delta_{i}\right\}$ lifts if only if $\delta_{i p}\left(\right.$ res $\left.R_{p}\right) \in k^{p}$ for each $i$ and $\delta_{i}\left(\right.$ res $\left.R_{p}\right)=0$ when $p \nmid i$.

CoROllaRy A. All higher derivations of $k$ lift to higher derivations of $R_{p}$ if and only if res $R_{p}=0$.

CoROllaRy B. All higher derivations of $k$ lift to higher derivations of $R_{p}$ if and only if $G_{D}=G_{S}$ and $p$ łexpo $R_{p}$.

II. Proofs and an application. Throughout this paper $R$ will denote an unramified $v$-subring of $R_{p}$ with $\left[R_{p}: R\right]=p, \pi$ a prime element of $R_{p}$ assumed to satisfy one of the equations $1.1,(1.2, \mathrm{t}) 1.3,, H\left(R, R_{p}\right)$ the set of higher derivations of $R$ into $R_{p}, H(k)$ the group of higher derivations of $k, k^{0}$ the maximal perfect subfield of $k$, and $v$ the exponential valuation on $R_{p}$.

Let $\left\{D_{i}\right\} \in H\left(R, R_{p}\right)$ and denote the unique extension of $\left\{D_{i}\right\}$ to the quotient field of $R_{p}$ by $\left\{D_{i}\right\}$. Note that $\left\{D_{i}\right\} \in H\left(R_{p}\right)$, the set of higher derivations of $R_{p}$, if and only if $D_{i}(\pi) \in(\pi)$ for each $i$. Let

$$
f(x)=x^{p}+p a_{p-1} x^{p-1}+\cdots+p a_{1} x+p a_{0}
$$

be the Eisenstein polynomial of $\pi$ over $R$. Let $(r, s)$ denote an ordered set of $r$ nonnegative integers whose sum is $s$ and let $|(r, s)|$ represent the largest integer in $(r, s)$. We let $\sum_{(q, s)} D\left(a_{1}, \cdots, a_{q}\right)$ denote the sum of all products $D_{i_{1}}\left(a_{1}\right) D_{i_{2}}\left(a_{2}\right) \cdots D_{i_{q}}\left(a_{q}\right)$ such that $i_{1}+\cdots+i_{q}$ $=s$ and $i_{j} \geqq 0$. We also let $f^{\prime}(x)$ and $f^{D} i(x)$ represent respectively the ordinary derivative of $f$ and the polynomial obtained by replacing each coefficient in $f$ with its image under $D_{i}$. With these conventions 
we may write the expression for $D_{i}(\pi)$ derived from $D_{i}(f(\pi))=0$ as follows:

$$
\begin{aligned}
\left(-f^{\prime}(\pi)\right) D_{i}(\pi)= & f_{i}{ }^{D}(\pi)+\sum_{(p, i) ;|(p, i)|<i} D(\pi, \cdots, \pi) \\
& +\sum_{j=1}^{p-1} p \sum_{(j+1, i),|(j+1, i)|<i} D\left(a_{j}, \pi, \cdots, \pi\right) .
\end{aligned}
$$

We list some observations.

I. $p \leqq v\left(f^{\prime}(\pi)\right) \leqq 2 p-1$.

II. If $a \in R$ then $D_{i}\left(a^{p^{n}}\right)=\left(D_{i / p^{n}}(a)\right)^{p^{n}} \bmod (\pi)^{p}$ where $D_{i / p^{n}}(a)=0$ if $p^{n} \nmid i$.

III. If $\pi^{p}+p u=0$ then $\bar{u}=\bar{a}_{0}$.

IV. If $\pi^{p}+p\left(1+\pi^{t} w\right)=0,1 \leqq t<p$, and $\bar{w} \neq 0$ then $\bar{w}=\bar{a}_{t}$ where $t$ is the least positive integer $i$ such that $p \nmid a_{i}$, and $a_{0}=1+p b_{0}$ for some $b_{0}$.

V. If $\pi^{p}+p\left(1+\pi^{p} w\right)=0, \quad \bar{w} \neq 0$ then $a_{0}=1+p b_{0}$ for some $b_{0}$, $\bar{w}=-\bar{b}_{0}$, and $p \mid a_{i}$ for all $i \geqq 1$.

VI. If $\pi^{p}+p\left(1+\pi^{p+1} w\right)=0$ then $a_{0}=1+p b_{0}$ for some $b_{0}$, $\bar{w}=\left[\left(-a_{1} / p\right)\right]-$, and $p \mid b_{0}$.

VII. If $1 \leqq$ expo $R_{p} \leqq p$ and $\left\{D_{i}\right\} \in H\left(R_{p}\right)$ then $D_{i}(\pi) \in(\pi)^{2}$ for each $i$.

If expo $R_{p}=p+1$ then $D_{1}(\pi) \in(\pi)^{3}$.

Let $\left\{D_{i}\right\} \in H\left(R_{p}\right),-f^{\prime}(\pi)=p \pi^{j-1} z, z$ a unit, $\pi A_{i}=D_{i}(\pi)$ when expo $R_{p}=0, \pi^{2} A_{i}=D_{i}(\pi)$ when $1 \leqq \operatorname{expo} R_{p} \leqq p$, and $\pi^{3} A_{i}=D_{i}(\pi)$ when expo $R_{p}=p+1$. We also write $a_{0}=1+p b_{0}$ when expo $R_{p} \neq 0$ and $p d_{1}=a_{1}$ when expo $R_{p}=p+1$.

2.3 Lemma. If expo $R_{p}=0$ and $\left\{D_{i}\right\} \in H\left(R_{p}\right)$ induces $\left\{\delta_{i}\right\} \in H(k)$ then res $R_{p}^{-1} \delta_{i p}\left(\right.$ res $\left.R_{p}\right) \in k^{p}$ for each $i$ and $\delta_{i}\left(\right.$ res $\left.R_{p}\right)=0$ when $p \nmid i$.

Proof. By 2.2,i we have $\left(p \pi^{j-1} z\right) D_{i}(\pi)=p D_{i}\left(a_{0}\right)+D_{i / p}(\pi)^{p}$, $\bmod (\pi)^{p+j}$ or $\left(\pi^{j-1} z\right) D_{i}(\pi)=D_{i}\left(a_{0}\right)-u A_{i / p}^{p}, \bmod (\pi)^{j}$ where $D_{i / p}(\pi)$ $=0=A_{i / p}$ if $p \nmid i$. Since $\bar{a}_{0}=\bar{u}=\operatorname{res} R_{p}$ and $D_{i}(\pi) \in(\pi)$ the result follows.

2.4 Lemma. If $1 \leqq$ expo $R_{p}<p$ and $\left\{D_{i}\right\} \in H\left(R_{p}\right)$ induces $\left\{\delta_{i}\right\}$ $\in H(k)$ then $\delta_{i}\left(\right.$ res $\left.R_{p}\right)=0$ for each $i$.

Proof. If res $R_{p} \neq 0$ then res $R_{p}=\bar{r}$ where $\bar{r}^{p^{n}}=\bar{a}_{j}, r \in R, \bar{r} \notin k^{p}$, expo $R_{p}=j$, and $v\left(f^{\prime}(\pi)\right)=p+(j-1)$. Using $(2.2, \mathrm{i})$ and II we get

$$
\begin{aligned}
\left(p \pi^{j-1} z\right) D_{i}(\pi) & =p D_{i}\left(a_{j}\right) \pi^{j}, & & \bmod (\pi)^{p+j+1} \\
& =p\left(D_{i / p^{n}}(r)\right)^{p^{n}} \pi^{j}, & & \bmod (\pi)^{p+j+1} .
\end{aligned}
$$

Again, since $D_{i}(\pi) \in(\pi)^{2}$ the result follows. 
2.5 Lemma. If expo $R_{p}=p$ and $\left\{D_{i}\right\} \in H\left(R_{p}\right)$ induces $\left\{\delta_{i}\right\} \in H(k)$ then $\delta_{i p}\left(\operatorname{res} R_{p}\right) \in k^{p}$ for each $i$ and $\delta_{i}\left(\right.$ res $\left.R_{p}\right)=0$ when $p \nmid i$.

Proof. We have res $R_{p}=-b_{0}$ and $\left(p \pi^{p-1} z\right) D_{i}(\pi)=p^{2} D_{i}\left(b_{0}\right)$ $+D_{i / p}(\pi)^{p}, \bmod \pi^{2 p+1}$. Thus $z D_{i}(\pi)=-u_{1}^{-1} \pi D_{i}\left(b_{0}\right)-u_{1} \pi A_{i / p}^{p}, \bmod$ $(\pi)^{2}$ where $\pi^{p}+p u_{1}=0$. But $D_{i}(\pi) \in(\pi)^{2}, \bar{u}_{1}=1$ and the proof is complete.

2.6 Lemma. If expo $R_{p}=p+1$ and $\left\{D_{i}\right\} \in H\left(R_{p}\right)$ induces $\left\{\delta_{i}\right\}$ $\in H(k)$ then $\delta_{i}\left(\right.$ res $\left.R_{p}\right)=0$ for each $i$.

Proof. Omitted.

We now show that if $\left\{\delta_{i}\right\} \in H(k)$ satisfies the conditions of the theorem then $\left\{\delta_{i}\right\}$ is induced. We assume $\left\{D_{i}\right\}_{i=1}^{n}$ is a finite higher derivation of $R$ into $R_{p}, A_{0}=1, f^{\prime}(\pi)$ and $A_{i}, i \geqq 1$ are as before. We list some observations which follow from $(2.2, \mathrm{i}), 1 \leqq i \leqq n$.

VIII. Let expo $R_{p}=0, v\left(f^{\prime}(\pi)\right)<2 p-1$ and $D_{i}(\pi) \in(\pi)$ for $i=1$, $\cdots, n-1$. If $D_{n}\left(a_{0}\right)-u A_{n / p}^{p}=\pi^{j} c_{n}$ then

$$
\begin{aligned}
\left(p \pi^{j-1} z\right) D_{n}(\pi)=p D_{n}\left(a_{j}\right) \pi^{j}+p D_{n}\left(a_{0}\right)+\left(D_{n / p}(\pi)\right)^{p} & \\
+p & \sum_{(j+1, n) ;|(j+1, n)|<n} D\left(a_{j} \pi \cdots \pi\right), \quad \bmod (\pi)^{p+j+1}
\end{aligned}
$$

where $v\left(f^{\prime}(\pi)\right)=p+(j-1)$. Thus if we write $\sum_{i_{m}<n} D_{i_{0}}\left(a_{j}\right) A_{i_{1}} \cdots A_{i_{j}}$ for the sum of all products $D_{i_{0}}\left(a_{j}\right) A_{i_{1}} \cdots A_{i_{j}}$ where $i_{0}+i_{1}$ $+\cdots+i_{j}=n, 0 \leqq i_{m}<n, m=0, \cdots, j$ then

$$
A_{n}=z^{-1}\left(D_{n}\left(a_{j}\right)+c_{n}+\sum_{i_{m}<n} D_{i_{0}}\left(a_{j}\right) A_{i_{1}} \cdots A_{i_{j}}\right), \bmod (\pi) .
$$

IX. Let expo $R_{p}=0, v\left(f^{\prime}(\pi)\right)=2 p-1$ and $D_{i}(\pi) \in(\pi)$ for $i=1$, $2, \cdots n-1$. If $D_{n}\left(a_{0}\right)-u A_{n / p}^{p}=\pi^{p} c_{n}$ then

$$
\left(p \pi^{p-1} z\right) D_{n}(\pi)=p D_{n}\left(a_{0}\right)+\sum_{(p, n) ;|(p, n)|<n} D(\pi, \cdots, \pi), \bmod (\pi)^{2 p+1} .
$$

Thus if in $\sum_{i_{m}<n} A_{i_{1}} \cdots A_{i_{p}}$ we also require that for some $m i_{m} \neq n / p$ we have

$$
A_{n}=z^{-1}\left[c_{n}+\frac{1}{p} \sum_{i_{m}<n} A_{i_{1}} \cdots A_{i_{p}}\right], \quad \bmod (\pi) .
$$

X. Let expo $R_{p}=p, \pi^{p}+p u_{1}=0$, and $D_{i}(\pi) \in(\pi)^{2}$ for $i=1,2$, $\cdots, n-1$. If $D_{n}\left(b_{0}\right)+u_{1}^{2} A_{n / p}^{p}=c_{n} \pi$ then

$$
\left(p \pi^{p-1} z\right) D_{n}(\pi)=p^{2} D_{n}\left(d_{1}\right) \pi+p^{2} D_{n}\left(b_{0}\right)+\left(D_{n / p}(\pi)\right)^{p}, \quad \bmod (\pi)^{2 p+2} .
$$

Thus $A_{n}=z^{-1}\left[-u_{1}^{-1} D_{n}\left(d_{1}\right)-u_{1}^{-1} c_{n}\right], \bmod (\pi)$. 
We may now proceed with the lifting of higher derivations of $k$ to $R_{p}$. The following theorem will be used repeatedly:

2.7 Theorem [3, Theorem 4, p. 38]. Let $\bar{S}$ be a p-basis for $k$ and let $S \subset R$ be a set of representatives of the elements of $\bar{S}$. If $I$ is the set of positive integers and $f$ is a mapping from $S \times I$ into $R_{p}$ then there is one and only one $\left\{D_{i}\right\} \in H\left(R, R_{p}\right)$ (finite higher derivation $\left\{D_{i}\right\}_{i=1}^{n}$ of length $n$ ) such that $D_{i}(s)=f(s, i)$ for all $s \in S$ and all $i \in I$ (all $s \in S$ and all $i=1,2, \cdots, n)$.

2.8 Lemma. Let expo $R_{p}=0$ and $v\left(f^{\prime}(\pi)\right)=p+(j-1)<2 p-1$. If $\left\{\delta_{i}\right\} \in H(k), \delta_{i}\left(\right.$ res $\left.R_{p}\right)=0$ when $p \nmid i$ and res $R_{p}^{-1} \delta_{i p}\left(\right.$ res $\left.R_{p}\right) \in k^{p}$ for each $i$, then $\left\{\delta_{i}\right\}$ lifts to a higher derivation of $R_{p}$.

Proof. Note that $\bar{a}_{0}=\bar{u}=$ res $R_{p}$. Let $B_{0}=1, B_{i} \in R_{p}$ so that $\bar{B}_{i}^{p}=\bar{u}^{-1} \delta_{i p}\left(\bar{a}_{0}\right)$, and $C_{i} \in R_{p}$ so that

$$
\bar{C}_{i}=\bar{z} \bar{B}_{i}-\delta_{i}\left(\bar{a}_{j}\right)-\sum_{i_{m}<i} \delta_{i_{0}}\left(\bar{a}_{j}\right) \bar{B}_{i_{1}} \cdots \bar{B}_{i_{j}} .
$$

Choose a $p$-basis $\bar{S}, \bar{a}_{0} \in \bar{S}$, for $k$ and let $S \subset R, a_{0} \in S$ be a set of representatives for $\bar{S}$. For $s \in S-\left\{a_{0}\right\}$ define $D_{1}$ so that $D_{1}$ will induce $\delta_{1}$. Let $D_{1}\left(a_{0}\right)=\pi^{j} C_{1}$. Then $D_{1}(\pi) \in(\pi)$ so $D_{1}$ extends to $R_{p}$. Also $\pi^{-1} D_{1}(\pi)=z^{-1}\left[D_{1}\left(a_{j}\right)+C_{1}\right], \bmod (\pi)$ or

$$
\bar{A}_{1}=\bar{z}^{-1}\left[\delta_{1}\left(\bar{a}_{j}\right)+\bar{z} \bar{B}_{1}-\delta_{1}\left(\bar{a}_{j}\right)\right]=\bar{B}_{1} \text {. }
$$

Suppose we have defined $\left\{D_{j}\right\}_{j=1}^{i-1}$ a finite higher derivation of $R$ into $R_{p}$ with the properties $D_{j}(\pi) \in(\pi), D_{j}$ induces $\delta_{j}$, and $\bar{A}_{j}=\bar{B}_{j}$. Define $D_{i}$ on $S-\left\{a_{0}\right\}$ so that $D_{i}$ induces $\delta_{i}$ and let $D_{i}\left(a_{0}\right)=\pi^{j} C_{i}+u A_{i / p}^{p}$. Then $\left[D_{i}\left(a_{0}\right)\right]-=\bar{u} \bar{A}_{i / p}^{p}=\bar{u} \bar{B}_{i / p}^{p}=\bar{u}\left(\bar{u}^{-1} \delta_{i}\left(\bar{a}_{0}\right)\right)=\delta_{i}\left(\bar{a}_{0}\right)$. Thus $D_{i}$ induces $\delta_{i}$. Also

$$
\begin{aligned}
\left(p \pi^{j-1} z\right) D_{i}(\pi)= & p D_{i}\left(a_{j}\right) \pi^{j}+p \pi^{j} C_{i}+u p A_{i / p}^{p}+\left(D_{i / p}(\pi)^{p}\right) \\
& +p \sum_{(j+1, i) ;|(j+1, i)|<i} D\left(a_{j}, \pi \cdots \pi\right), \bmod (\pi)^{p+j+1}
\end{aligned}
$$

so $D_{i}(\pi) \in(\pi)$. Using this equation we have

$$
\begin{aligned}
\bar{A}_{i}=\bar{z}^{-1}\left[\delta_{i}\left(\bar{a}_{j}\right)+\bar{z} \bar{B}_{i}-\delta_{i}\left(\bar{a}_{j}\right)-\sum_{i_{m}<i} \delta_{i_{0}}\left(\bar{a}_{j}\right) \bar{B}_{i_{1}} \cdots \bar{B}_{i_{j}}\right. \\
\\
\left.\quad+\sum_{i_{m}<i} \delta_{i_{0}}\left(\bar{a}_{j}\right) \bar{A}_{i_{1}} \cdots \bar{A}_{i_{j}}\right]=\bar{B}_{i} .
\end{aligned}
$$

The lemma follows from 2.7 and mathematical induction. 
2.9 Lemma. Let expo $R_{p}=0$ and $v\left(f^{\prime}(\pi)\right)=2 p-1$. If $\left\{\delta_{i}\right\} \in H(k)$, $\delta_{i}\left(\right.$ res $\left.R_{p}\right)=0$ when $p \nmid i$, and res $R_{p}^{-1} \delta_{i p}$ (res $\left.R_{p}\right) \in k^{p}$ for each $i$ then $\left\{\delta_{i}\right\}$ lifts to a higher derivation of $R_{p}$.

Proof. Let $B_{i} \in R_{p}$ be such that $\bar{B}_{i}^{p}=\bar{u}^{-1} \delta_{i p}\left(\bar{a}_{0}\right)$ and $C_{i}=z B_{i}$ $-p^{-1} \sum_{i_{m}<i} B_{i_{1}} \cdots B_{i_{p}}$, where in the summation, $\sum_{i_{m}<i} B_{i_{1}} \cdots B_{i_{p}}$, we also require that for some $m=1, \cdots, p i_{m} \neq i / p$ (as in IX). Choose a $p$-basis $\bar{S}, \bar{a}_{0} \in \bar{S}$, for $k$ and let $S \subset R, a_{0} \in S$ be a set of representatives for $S$. Define $D_{1}$ on $S-\left\{a_{0}\right\}$ so that $D_{1}$ will induce $\delta_{1}$. Let $D_{1}\left(a_{0}\right)=\pi^{p} C_{1}$. Then $D_{1}$ induces $\delta_{1}, D_{1}(\pi) \in(\pi)$, and $A_{1}=B_{1}, \bmod (\pi)$. Suppose $\left\{D_{j}\right\}_{j=1}^{i-1}$ is a finite higher derivation of $R$ into $R_{p}$ with $D_{j}$ inducing $\delta_{j}, D_{j}(\pi) \in(\pi)$, and $A_{j}=B_{j}, \bmod (\pi)$. Define $D_{i}$ on $S-\left\{a_{0}\right\}$ so that $D_{i}$ induces $\delta_{i}$ and let $D_{i}\left(a_{0}\right)-u A_{i / p}^{p}=\pi^{p} C_{i}$. Then $D_{i}$ induces $\delta_{i}, D_{i}(\pi) \in(\pi)$, and by IX

$$
\begin{aligned}
A_{i} & =z^{-1}\left(C_{i}+\frac{1}{p} \sum_{i_{m}<i} A_{i_{1}} \cdots A_{i_{p}}\right), & \bmod (\pi) \\
& =z^{-1}\left(z B_{i}-\frac{1}{p} \sum_{i_{m}<i} B_{i_{1}} \cdots B_{i_{p}}+\frac{1}{p} \sum_{i_{m}<i} A_{i_{1}} \cdots A_{i_{p}}\right), & \bmod (\pi) .
\end{aligned}
$$

Thus, again $\bar{A}_{i}=\bar{B}_{i}$ and the proof is complete.

2.10 LemmA. Let $1 \leqq$ expo $R_{p}=j<p$. If $\left\{\delta_{i}\right\} \in H(k)$ and $\delta_{i}$ (res $\left.R_{p}\right)=0$ for each $i$ then $\left\{\bar{\delta}_{i}\right\}$ lifts to a higher derivation of $R_{p}$.

PROOF. If res $R_{p} \neq 0$ then res $R_{p}=\bar{r}$ where $\bar{r}^{n}=\overline{a_{j}}, r \in R$, and $\bar{r} \notin k^{p}$. Let $\bar{S}, \bar{r} \in \bar{S}$, be a $p$-basis for $k$ and $S \subset R, r \in S$, a set of representatives for $\bar{S}$. For each $i$ define $D_{i}$ on $S-\{r\}$ so that $D_{i}$ induces $\delta_{i}$. Let $D_{i}(r)=0$ for all $i$. Then $\left\{D_{i}\right\}$ induces $\left\{\delta_{i}\right\}$ and by checking $(2.2, i)$ $\left\{D_{i}\right\}$ extends to $R_{p}$. If res $R_{p}=0$ choose any $p$-basis $\bar{S}$ for $k$ and $S \subset R$ a set of representatives for $\bar{S}$. Define $\left\{D_{i}\right\}$ on $S$ so that $D_{i}$ induces $\delta_{i}$ for each $i$. Then 2.2 , i and II imply $\left\{D_{i}\right\}$ extends to $R_{p}$.

2.11 Lemma. Let expo $R_{p}=p$ and $\pi^{p}=p u_{1}=0$. If $\left\{\delta_{i}\right\} \in H(k)$, $\delta_{i p}\left(\right.$ res $\left.R_{p}\right) \in k^{p}$ for each $i$, and $\delta_{i}\left(\right.$ res $\left.R_{p}\right)=0$ when $p \nmid i$, then $\left\{\delta_{i}\right\}$ lifts to a higher derivation of $R_{p}$.

Proof. By $V a_{0}=1+p b_{0}$ and res $R_{p}=-\bar{b}_{0}$. Let $\bar{B}_{i}^{p}=-\delta_{i p}\left(\bar{b}_{0}\right)$ and $\bar{C}_{i}=-\delta_{i}\left(\bar{d}_{1}\right)-\bar{z} \bar{B}_{i}$, where again $a_{1}=p d_{1}$. Choose a $p$-basis $\bar{S}, \bar{b}_{0} \in \bar{S}$, for $k$ and let $S \subset R, b_{0} \in S$, be a set of representatives for $\bar{S}$. Define $D_{1}$ on $S-\left\{b_{0}\right\}$ so that $D_{1}$ induces $\delta_{1}$ and let $D_{1}\left(b_{0}\right)=\pi C_{1}$. Then $D_{1}$ induces $\delta_{1}, D_{1}(\pi) \in(\pi)^{2}$, and $\bar{A}_{1}=\bar{B}_{1}$. Suppose we have defined $\left\{D_{j}\right\}_{j=1}^{i-1}$, a finite higher derivation of $R$ into $R_{p}$, with $D_{j}$ inducing $\delta_{j}$, $D_{j}(\pi) \in(\pi)^{2}$ and $\bar{A}_{j}=\bar{B}_{j}$. Define $D_{i}$ on $S-\left\{b_{0}\right\}$ so that $D_{i}$ induces 
$\delta_{i}$ and let $D_{i}\left(b_{0}\right)+u_{1}^{2} A_{i / p}^{p}=\pi C_{i}$. Then $D_{i}$ induces $\delta_{i}, D_{i}(\pi) \in(\pi)^{2}$, and by $\mathrm{X} \bar{A}_{i}=\bar{B}_{i}$.

2.12 Lemma. Let expo $R_{p}=p+1$. If $\left\{\delta_{i}\right\} \in H(k)$ and $\delta_{i}\left(\right.$ res $\left.R_{p}\right)=0$ for each $i$ then $\left\{\delta_{i}\right\}$ lifts to a higher derivation of $R_{p}$.

Proof. Omitted.

In view of the fact that higher derivations of $k$ are completely determined by their action on a $p$-basis for $k$ corollary $\mathrm{A}$ is clear. Corollary B follows from [5].

Let $S$ be a ring and $\tau$ the natural map of $S[[x]]$ onto $S$. The inertial embedding group of $S$ is the group of all automorphisms $\eta$ of $S[[x]]$ such that $\tau \eta(a)=a$, for each $a \in S$, and $\eta(x)=x$. The higher derivation $\left\{D_{i}\right\}$ of $S$ determines an (inertial) embedding $\eta$ of $S$ where $\eta(a)=a+\sum_{i=1}^{\infty} D_{i}(a) x_{i}$ for $a \in S$ and $\eta(x)=x$. Conversely an embedding of $S$ determines a higher derivation of $S$. The correspondence is an isomorphism of the higher derivation group of $S$ onto the inertial embedding group of $S$. An application of our theorem will identity the subgroup of the embedding group of $k$ consisting of those embeddings which are induced by embeddings of $R_{p}$.

\section{REFERENCES}

1. N. Heerema, Embeddings of a p-adic field and its residue field in their power series rings, Proc. Amer. Math. Soc. 14 (1963), 574-580.

2. - Derivations and intertial automorphisms of complete regular local rings, Amer. J. Math. 88 (1966), 33-42.

3. - Convergent higher derivations on local rings, Trans. Amer. Math. Soc. 132 (1968), 31-44.

4. - Intertial automorphisms of a class of wildly ramified v-rings, Trans. Amer. Math. Soc. 132 (1968), 45-54.

5. M. Heinzer, Strongly convergent derivation automorphisms on a class of wildly ramified v-rings, Duke Math. J. (to appear).

6. J. Neggers, Derivations on p-adic fields, Trans. Amer. Math. Soc. 115 (1965), 496-504.

7. E. Wishart, Higher derivations on $\bar{p}$-adic fields, Dissertation, Florida State University, Tallahassee, Fla., 1965.

Florida State University 